Check for updates

Cite this: Phys. Chem. Chem. Phys., 2021, 23, 7696

Received 18th May 2020, Accepted 30th June 2020

DOI: $10.1039 / \mathrm{d} 0 \mathrm{cp} 02698 \mathrm{k}$

rsc.li/pccp

\title{
CO adsorption on the calcite(10.4) surface: a combined experimental and theoretical study
}

\author{
Tahereh Mohammadi Hafshejani, $\dagger^{a}$ Weijia Wang, $\dagger^{a}$ Jonas Heggemann, \\ Alexei Nefedov, (D) a Stefan Heissler, ${ }^{a}$ Yuemin Wang, (D) ${ }^{a}$ Philipp Rahe, (D) ${ }^{b}$ \\ Peter Thissen (D) ${ }^{a}$ and Christof Wöll (D) *a
}

\begin{abstract}
Detailed information on structural, chemical, and physical properties of natural cleaved (10.4) calcite surfaces was obtained by a combined atomic force microscopy (AFM) and infrared (IR) study using CO as a probe molecule under ultrahigh vacuum (UHV) conditions. The structural quality of the surfaces was determined using non-contact AFM (NC-AFM), which also allowed assigning the adsorption site of CO molecules. Vibrational frequencies of adsorbed $\mathrm{CO}$ species were determined by polarization-resolved infrared reflection absorption spectroscopy (IRRAS). At low exposures, adsorption of $\mathrm{CO}$ on the freshly cleaved (10.4) calcite surface at a temperature of $62 \mathrm{~K}$ led to the occurrence of a single $\mathrm{C}$-O vibrational band located at $2175.8 \mathrm{~cm}^{-1}$, blue-shifted with respect to the gas phase value. For larger exposures, a slight, coverage-induced redshift was observed, leading to a frequency of $2173.4 \mathrm{~cm}^{-1}$ for a full monolayer. The width of the vibrational bands is extremely small, providing strong evidence that the cleaved calcite surface is well-defined with only one $\mathrm{CO}$ adsorption site. A quantitative analysis of the IRRA spectra recorded at different surface temperatures revealed a $\mathrm{CO}$ binding energy of $-0.31 \mathrm{eV}$. NCAFM data acquired at $5 \mathrm{~K}$ for sub-monolayer $\mathrm{CO}$ coverage reveal single molecules imaged as depressions at the position of the protruding surface features, in agreement with the IRRAS results. Since there are no previous experimental data of this type, the interpretation of the results was aided by employing density functional theory calculations to determine adsorption geometries, binding energies, and vibrational frequencies of carbon monoxide on the (10.4) calcite surface. It was found that the preferred geometry of $\mathrm{CO}$ on this surface is adsorption on top of calcium in a slightly tilted orientation. With increased coverage, the binding energy shows a small decrease, revealing the presence of repulsive adsorbate-adsorbate interactions.
\end{abstract}

\section{Introduction}

Calcium carbonate $\left(\mathrm{CaCO}_{3}\right)$ is a widely occurring mineral in nature, constituting a substantial part of the Earth's crust. ${ }^{1}$ The most stable polymorph of $\mathrm{CaCO}_{3}$ is calcite, which is a common component of all-natural rock types. ${ }^{2}$ This ionic compound is also abounding in living organisms, is incorporated into bones, teeth, as well as in shells of mussels and plays an important role in biomineralization. ${ }^{2,3}$ Calcite has been used as construction material, for agricultural soil treatment, as sorbent, for pharmaceutical applications, ${ }^{4}$ and has been integrated in optical devices utilizing its birefringence property. ${ }^{5}$ As the largest

\footnotetext{
${ }^{a}$ Karlsruher Institut für Technologie (KIT), Institut für Funktionelle Grenzflächen (IFG), Hermann-von-Helmholtz-Platz 1, 76344 Eggenstein-Leopoldshafen, Germany. E-mail: christof.woell@kit.edu

${ }^{b}$ Fachbereich Physik, Universität Osnabrück, Barbarastrasse 7, 49076 Osnabrück, Germany

$\dagger$ These authors contributed equally to this work.
}

carbon dioxide repository, this carbonate also played a significant role for the composition of the planet's atmosphere. ${ }^{6}$ Knowledge about calcite surfaces, their detailed structure, and chemical properties are thus highly relevant.

Calcite is an ionic crystal consisting of calcium cations $\left(\mathrm{Ca}^{2+}\right)$ and carbonate $\left(\mathrm{CO}_{3}{ }^{2-}\right)$ anions, which are held together through ionic bonding. ${ }^{7}$ The structure is trigonal (rhombohedral holohedral $\overline{3} 2 / m$ ) with a $R \overline{3} c$ space group. ${ }^{7 c}$ Cleavage is easiest for the thermodynamically most stable calcite(10.4) surface with a surface energy calculated around $0.59 \mathrm{~J} \mathrm{~m}^{-2}$. The calcite(10.4) surface is furthermore nonpolar, namely a type I surface according to Tasker's classification, and thus bears zero charge $(q=0)$ and zero dipole moment $(\mu=0){ }^{9}$ Accordingly, most previous surface studies reported for this material focused on this surface. The calcite(10.4) surface (a structural model is reproduced in Fig. 1) contains a total of two calcium atoms and two carbonate groups within the $(1 \times 1)$ unit cell (with a unit cell size of $5.0 \times 8.1 \AA^{2}$ ), both are present in the top layer. Note that the two carbonate groups are rotated 
(a)
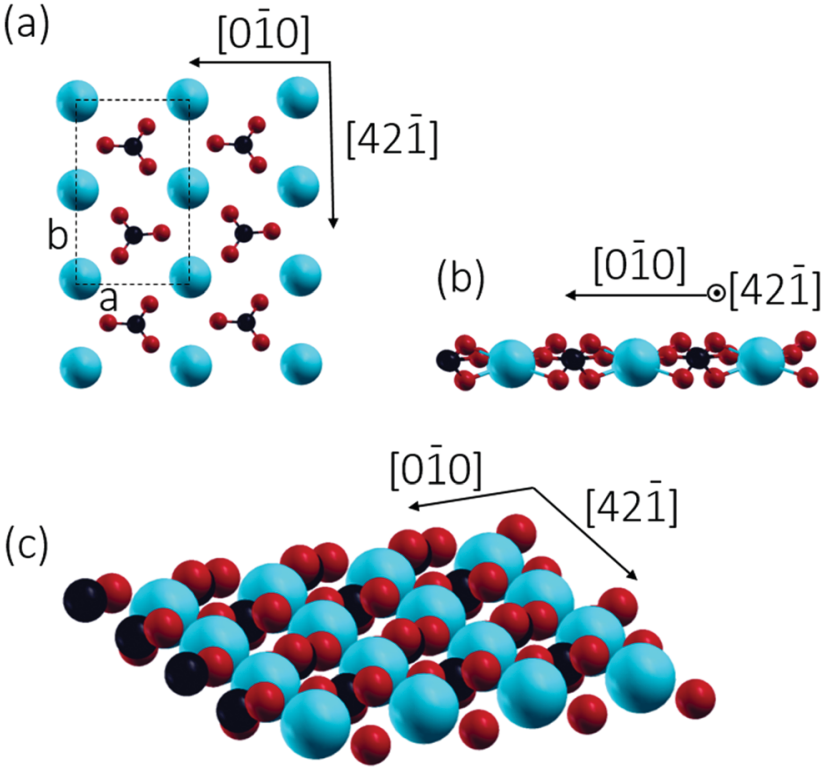

Fig. 1 Top (a) and side (b) views of the $(1 \times 1)$ calcite $(10.4)$ surface model $(a=5.0 \AA$ and $b=8.1 \AA$ ). Bottom image (c) shows the atoms exposed the surface, revealing that the oxygens are located at two different but equivalent positions. (red: oxygen, black: carbon, blue: calcium).

with respect to each other and, as a consequence, the upmost oxygen atom points either to the left or to the right along the [42ī] direction. ${ }^{7 b, 10}$

The microscopic nature of the calcite(10.4) surface has intensively been studied by various experimental ${ }^{10,11}$ and theoretical methods. ${ }^{12}$ Two deviations from the bulk-truncated $(1 \times 1)$ structure have been reported with their origins and geometry still being under discussion. First, a loss of the $p g$ surface symmetry (also referred to as "row-pairing") has been observed within atomic force microscopy (AFM) data, ${ }^{10,11,13}$ yet, this surface relaxation can also be explained by an asymmetric tip probing an unrelaxed surface. Second, the existence of a $(2 \times 1)$ reconstruction has been proposed on the basis of low energy electron diffraction (LEED) ${ }^{11 d}$ and AFM data. ${ }^{11 b, 14}$ This reconstruction is characterised by a rectangular supercell with dimensions of $10.0 \AA$ and $8.1 \AA$ oriented along the [010] and [42î] directions, respectively.

Here, the surface structure of the UHV-cleaved calcite(10.4) surface is investigated by polarization-resolved infrared reflection absorption spectroscopy (IRRAS) using the surface-ligand IR (SLIR) spectroscopy approach, ${ }^{15}$ with $\mathrm{CO}$ as the probe molecule, and complemented by non-contact atomic force microscopy (NC-AFM) experiments and density functional theory (DFT) calculations. We would like to point out that there are no previous IRRAS studies of adsorbates on well-defined surfaces of macroscopic calcite single crystals available. This lack of experimental data is due to technical problems in recording IR-data using reflection geometries for dielectric substrates, which could only recently be overcome. ${ }^{15,16}$ The IRRAS results of $\mathrm{CO}$ on calcite(10.4) surfaces obtained at low temperature show only one sharp absorption band, providing strong evidence that this surface exhibits only one type of $\mathrm{Ca}^{2+}$ binding site. To our knowledge, these are the first IRRAS data for any adsorbates bound to a calcite single crystal substrate.

\section{Methods}

\section{Infrared reflection absorption spectroscopy}

All IRRAS experiments were performed in a multi-technique UHV apparatus (Prevac, Poland). A more comprehensive description of the experimental setup can be found elsewhere. ${ }^{16}$ Large blocks of calcite(10.4) single crystal $\left(8 \times 8 \times 12 \mathrm{~mm}^{3}\right)$ with optical quality were purchased from Korth Kristalle GmbH (Altenholz, Germany). The blocks were cut into small pieces in atmosphere before attaching them to the sample holder. Cleavage of the calcite single crystal in the UHV chamber was carried out using a scalpel blade attached to a wobble stick. A photograph of the sample holder with a piece of a calcite single crystal is shown in Fig. 2. The central part of the base plate can be rotated in clockwise and anticlockwise directions using the wobble stick inside the UHV chamber, so that the azimuthal orientation of the specimen can be changed. After the single crystal was introduced into the UHV system, it was first briefly annealed before cleavage. After cleavage the samples were quickly transferred to the IRRAS chamber. The IRRAS data were acquired using polarized infrared light at a grazing incidence angle $\left(80^{\circ}\right)$ with a spectral resolution of $1 \mathrm{~cm}^{-1}$. The base pressure of the UHV chamber amounted to $4.0 \times 10^{-11} \mathrm{mbar}$. Dosing of $\mathrm{CO}$ was performed using a capillary tube ending in front of the sample with the temperature of the sample held at $62 \mathrm{~K}$. The temperature of the sample was monitored by a K-type thermocouple that was attached to the edge of the sample holder. An exposure of one Langmuir (L) corresponds to $1.33 \times 10^{-6} \mathrm{mbar} \mathrm{s}$.

\section{Non-contact atomic force microscopy (NC-AFM)}

Sample preparation and NC-AFM experiments were performed under ultra-high vacuum conditions in a system separate from the IRRAS setup. Calcite crystals (Korth Kristalle, Altenholz, Germany) were cleaved in-situ to produce a (10.4) surface by

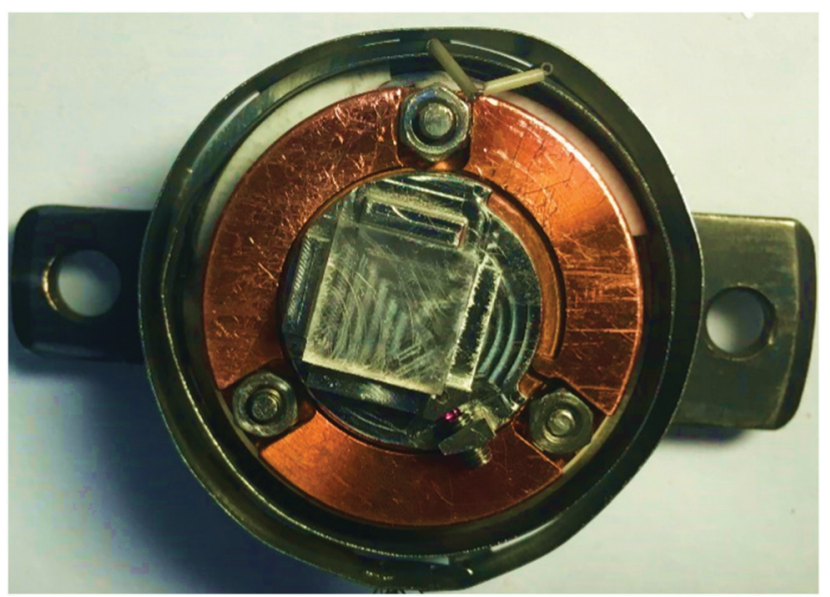

Fig. 2 Photograph of a calcite $\left(\mathrm{CaCO}_{3}\right)$ single crystal mounted in the sample holder (designed for UHV cleavage) with the (10.4) surface pointing up. Size of the base plate of the sample holder is $8 \times 8 \mathrm{~mm}^{2}$. 
using a scalpel blade attached to a wobble stick. ${ }^{17}$ The crystal was mounted on a double sample holder supporting an additional $\mathrm{Ag}(111)$ surface for tip preparation and was heated for $1.5 \mathrm{~h}$ to about $440 \mathrm{~K}$ to remove residual charges. ${ }^{18}$ NC-AFM experiments were performed with an LT qPlus gen.III microscope (ScientaOmicron, Taunusstein, Germany) operated by a MATRIX controller and using W-tips attached to qPlus sensors ${ }^{19}$ as supplied by the manufacturer. Tips were prepared by common scanning tunnelling microscopy strategies on the $\mathrm{Ag}(111)$ surface mounted nearby the calcite sample. CO was dosed directly into the scan head $\left(T_{\text {sample }}<10 \mathrm{~K}\right.$ while opening the shields) at background pressures around $p \sim 5 \times 10^{-8}$ mbar. Metal tips were partly terminated with CO molecules for improving the imaging contrast. ${ }^{20}$ The surface orientation has been determined ex-situ after the NC-AFM experiments by optically identifying the orientation of the ordinary and extraordinary ray of the birefringence. ${ }^{21}$

\section{Computational details}

DFT calculations were performed using the generalized gradient approximation (GGA) and the PBE functional, as implemented in the Vienna $a b$ initio simulation package (VASP). ${ }^{22}$ The electron-ion interaction was described within the projector-augmented wave (PAW) scheme. $^{23}$ The electronic wave functions were expanded into plane waves up to a kinetic energy of $400 \mathrm{eV}$.

The calculations reported here were carried out for a slab (thickness of 4 layers) exposing a (10.4) surface with a $(1 \times 1)$ unit cell and containing a total number of 40 atoms (Ca: 8, C: 8, O: 24), see also Fig. 1. This model is similar to that used in earlier studies. $^{8 b, 24}$ A vacuum region of $20 \AA$ was found to be large enough to avoid interactions between the surface and adsorbed molecules and their replica images. We define coverage as the ratio of the number of $\mathrm{CO}$ molecules to the number of surface $\mathrm{Ca}$ ions. All degrees of freedom, except the bottom layer, were allowed to relax until the forces on the atoms were below $1 \mathrm{meV} \AA^{-1}$. The optimization of the atomic coordinates (and unit cell size for the bulk materials) was performed through a conjugate gradient technique, which utilizes the total energy and the HellmannFeynman forces on the atoms (and stresses on the unit cell). The structures were considered to be fully relaxed when the forces on the atoms were smaller than $1 \mathrm{meV} \AA^{-1}$. The Brillouin zone integration was performed using a $4 \times 4 \times 1$ mesh within the Monkhorst-Pack scheme. ${ }^{25}$ Vibrational eigenmodes and frequencies were calculated by the force-constant approach. The start geometry for the optimization was a CO molecule (bond length $1.14 \AA$ ) positioned on top of $\mathrm{Ca}^{2+}$ in an upright orientation.

The surface energy is defined as the difference in total energy between the bulk and the surface per unit area and, for a stoichiometric surface, can be obtained by the following equation: ${ }^{26}$

$$
E_{\text {surf }}=\left(E_{\text {slab }}-N E_{\text {bulk }}\right) / 2 A
$$

where $E_{\text {slab }}$ is the total energy of the surface, $E_{\text {bulk }}$ is the bulk energy, $N$ is the number of unit cells used to build the slab and $A$ is the surface area. The obtained surface energy from our calculations was $0.50 \mathrm{~J} \mathrm{~m}^{-2}$ which is quite close to previous calculations. ${ }^{8 b, 27}$

The adsorption energy $\left(E_{\text {ads }}\right)$ can be calculated as a function of the coverage of the CO molecules: ${ }^{28}$

$$
E_{\mathrm{ads}}=\frac{E_{n}-E_{0}-n E_{\mathrm{CO}}}{n}
$$

where $E_{n}$ is the energy of the surface containing $n$ molecules, $E_{\mathrm{CO}}$ is the energy of the gas-phase molecule, and $E_{0}$ is the energy of the clean slab.

\section{Results and discussion}

The polarization-resolved IRRAS data shown in Fig. 3a were recorded for p-polarized IR light incident along the surface after varying exposures of the freshly cleaved calcite(10.4) surface to $\mathrm{CO}$ at a substrate temperature of $62 \mathrm{~K}$. For an exposure of $0.05 \mathrm{~L}$, an asymmetric single band located at $2175.8 \mathrm{~cm}^{-1}$ is observed, which is assigned to the stretching vibration of $\mathrm{CO}$ molecules bound with the $\mathrm{C}$ atom to $\mathrm{Ca}^{2+}$-cations at the calcite surface. When increasing the exposure to $0.1 \mathrm{~L}$, the band increased in intensity and, at the same time, shifted slightly to lower frequency, $2174.4 \mathrm{~cm}^{-1}$. With increasing exposures, the lowering of frequency continued until a value of $2173.4 \mathrm{~cm}^{-1}$ was reached. For exposures higher than $2.0 \mathrm{~L}$ no further increases of the band intensity or changes of the band shape were observed, indicating that the calcite(10.4) surface was saturated with CO and no multilayer formation occurred at this substrate temperature.

The CO vibration was also observed in IRRAS data recorded with s-polarized light (Fig. 3b, bottom). However, the intensity is much smaller, by an order of magnitude, compared to that excited by p-polarized light (Fig. 3b, top). The occurrence of a vibrational feature also for s-polarization reveals that there must be a static or dynamic tilt angle, because only vibrational modes with a component of their transition dipole moment (TDM) orientated parallel to the surface can be excited by s-polarized light. ${ }^{16}$
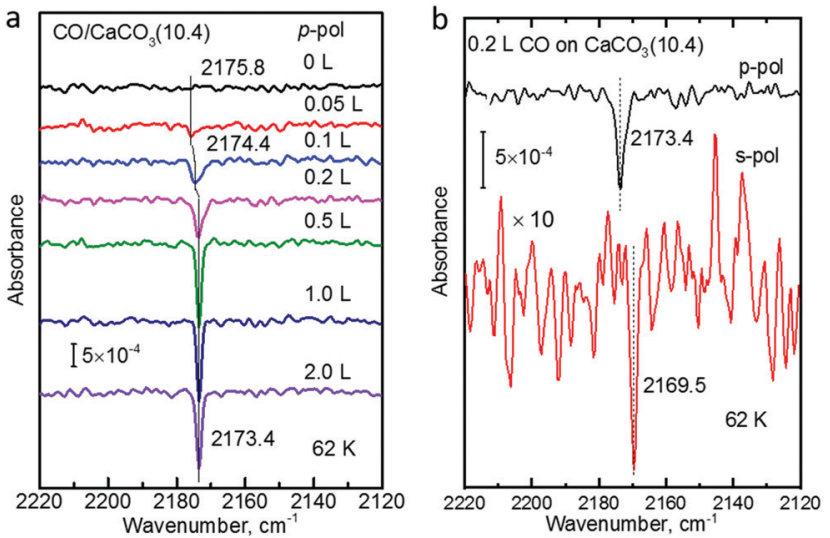

Fig. 3 (a) IRRAS spectra with $\mathrm{p}$-polarized light for $\mathrm{CO}$ adsorbed onto the calcite(10.4) surface at $62 \mathrm{~K}$ with increasing exposures. (b) IRRAS spectra with $\mathrm{p}$-polarized light (top) and s-polarized light (bottom) for $0.2 \mathrm{~L} \mathrm{CO}$ adsorbed onto the calcite(10.4) surface at $62 \mathrm{~K}$. 

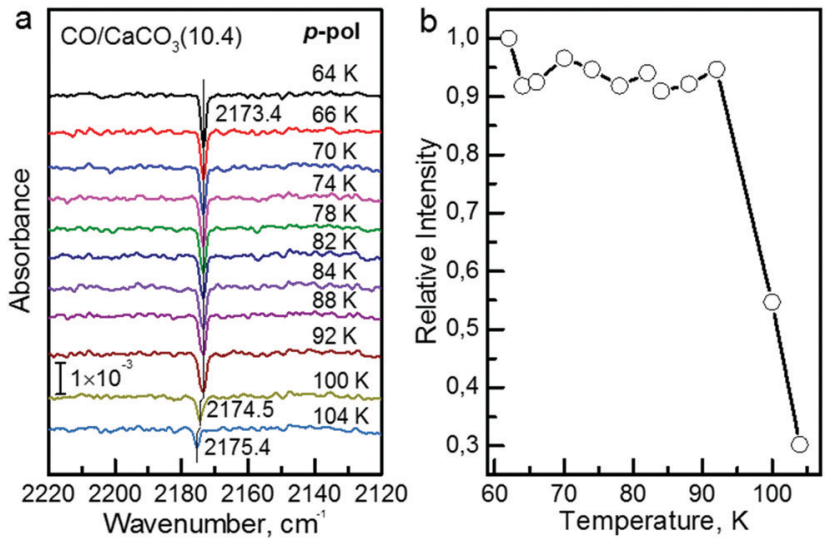

Fig. 4 (a) IRRAS spectra for $2.0 \mathrm{~L}$ of $\mathrm{CO}$ adsorbed onto calcite(10.4) surfaces at $T=62 \mathrm{~K}$ and after annealing to indicated temperatures. (b) Integrated intensity evolution of the spectra as a function of temperature.

In order to determine the binding energy of the CO adlayers we recorded IRRAS-data after heating to different temperatures, see Fig. 4a. In Fig. 4b we present the variation of the integrated band intensities as a function of surface temperature. The position (Fig. 4a) and the intensity (Fig. 4b) of the $\mathrm{CO}-\mathrm{Ca}^{2+}$ band stays constant for temperatures below $92 \mathrm{~K}$. Upon further annealing to $100 \mathrm{~K}$, CO starts to desorb from the surface, yielding a decrease in band intensity accompanied by a slight blue shift of the vibrational frequency. A simple quantitative analysis of the IR band intensity evolution, using the Redhead equation, ${ }^{29}$ yields a binding energy of $-0.31 \mathrm{eV}$ for $\mathrm{CO}$ on the calcite(10.4) surface.

Next, we recorded AFM micrographs to investigate the adsorption position and $\mathrm{CO}$ arrangement after dosing $\mathrm{CO}$ into the cold scan head on calcite(10.4) for three different total times of $25 \mathrm{~s}, 50 \mathrm{~s}$, and $150 \mathrm{~s}$, resulting in a coverage of about $0.006,0.013$ and 0.067 monolayers (ML), respectively. $1 \mathrm{ML}$ is defined as a coverage of two CO molecules per $(1 \times 1)$ unit cell. Note that the pressure was slightly higher in the third experiment. The corresponding results are shown in Fig. 5. The calcite surface structure is in all images apparent in the form of four bright protrusions per $(2 \times 1)$ unit cell, having unit cell dimensions of $10 \times 8.1 \AA^{2}$ as indicated by white rectangles. The contrast in Fig. 5a, b and d represents a staggered (zig-zag) structure along the $[42 \overline{1}]$ direction, which can be related to the different orientation of the two carbonate groups within the surface unit cell. Additionally, the $(2 \times 1)$ reconstruction is visible as a modulation along the [010] direction of the dark rows in all micrographs. As no row-pairing reconstruction is apparent, the $p g$ surface symmetry is maintained.

Individual dark depressions, which are not observed on pristine calcite(10.4) surfaces, are visible and identified as single CO molecules (examples are marked by white arrows in Fig. 5). These depressions are well separated from each other, but are often manipulated while scanning (see Fig. 5c). We did not observe the formation of clusters or adsorbate islands. As apparent from the detailed micrograph in Fig. 5d, the molecules are located at the position of calcite surface features imaged bright in NC-AFM. Despite the sharp single band
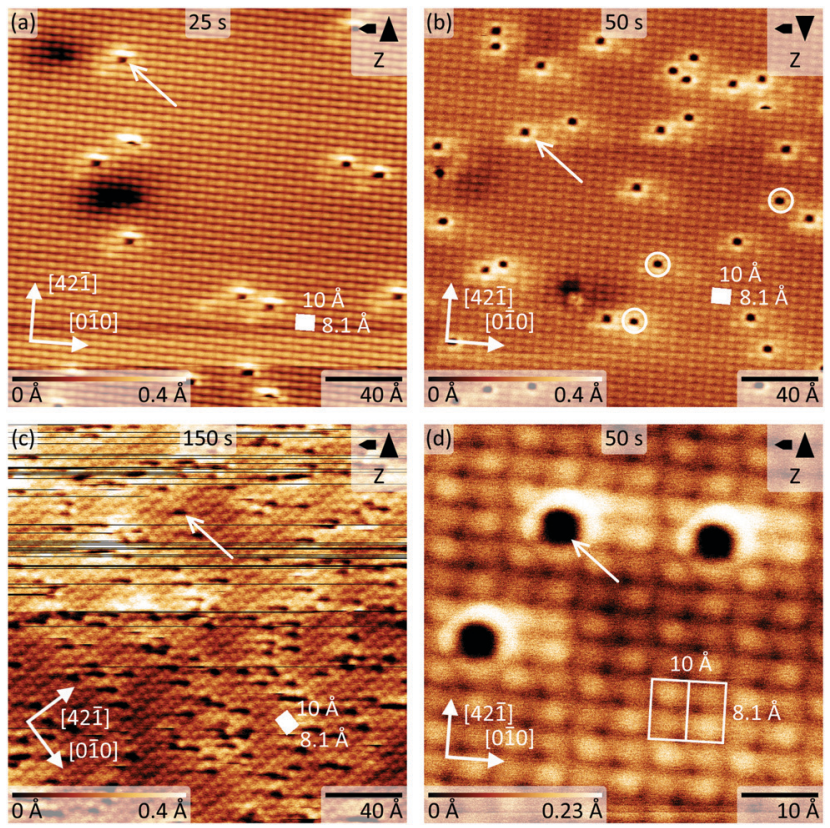

Fig. 5 Imaging single CO molecules on calcite(10.4) with NC-AFM at $5 \mathrm{~K}$ using total deposition times of (a) $25 \mathrm{~s}$, (b and d) $50 \mathrm{~s}$, and (c) $150 \mathrm{~s}$. Data in ( $b$ and $d$ ) were acquired after dosing further $25 \mathrm{~s}$ onto the sample presented in (a). Data in (c) are acquired after dosing for $25 \mathrm{~s}$ and additional 125 s. Single CO molecules are marked by an arrow in all figures, molecules slightly off-center with white circles in (b). The $(2 \times 1)$ unit cell is marked by white rectangles in all micrographs.

measured with IRRAS, we also observe molecules positioned slightly off-center the bright calcite surface feature (examples are marked by white circles in Fig. 5b). However, the difference between these orientations might be thermally averaged at the IRRAS measurement temperature of $62 \mathrm{~K}$, while the barrier is too high at the NC-AFM imaging temperature of $5 \mathrm{~K}$. Based on the IRRAS results we can furthermore conclude that the bright protrusions in these NC-AFM micrographs are representing the locations of the surface $\mathrm{Ca}^{2+}$ cations as the $\mathrm{CO}$ molecules are located at these positions. Two possibilities including a "positive" or "negative potential nanotip" imaging model were suggested for NC-AFM measurements of calcite(10.4) surfaces before. ${ }^{30}$ The imaging was explained to be dominated by electrostatic interactions, where either the oxygen or calcium sublattices are imaged as "bright" due to attraction with a positive or negative ion located at the tip apex, respectively. ${ }^{30}$ Thus, we conclude that the data herein were acquired with a probing tip with a negative termination.

Finally, we studied two different $\mathrm{CO} /$ calcite configurations by DFT, namely (i) a single CO molecule adsorbed within the $(1 \times 1)$ cell, corresponding to a coverage of $0.5 \mathrm{ML}$, and (ii) two CO molecules adsorbed within the $(1 \times 1)$ cell, corresponding to a complete monolayer coverage $(1.0 \mathrm{ML})$. In all cases, $\mathrm{CO}$ was adsorbed on-top of a surface Ca site and initially positioned perpendicular to the surface with the carbon pointing downwards. This geometry corresponds to the common coordination of CO on metals and metal oxides. ${ }^{31}$ Optimization with DFT yielded a tilt of $14.42^{\circ}$ for $0.5 \mathrm{ML}$ and tilts of $14.57^{\circ}$ and $15.14^{\circ}$ 


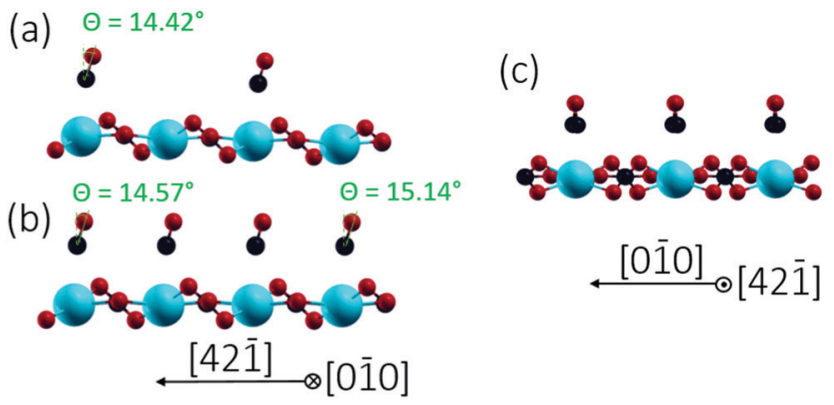

Fig. 6 Side views of CO/calcite(10.4) after energy minimization for two different $\mathrm{CO}$ coverages of $0.5 \mathrm{ML}$ (a) and 1.0 ML (b and c). CO tilt angles are included.

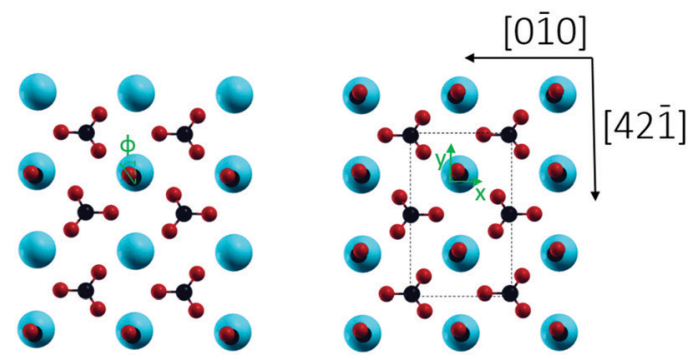

Fig. 7 Top view of $\mathrm{CaCO}_{3}$ surface unit cell with one carbon monoxide molecule (0.5 ML coverage, left) and a complete monolayer of $\mathrm{CO}$ (right) on the energetically favored adsorption position. $\Phi$ represents the azimuthal angle of adsorbed $\mathrm{CO}$ after their optimized configurations.

Table 1 Parameter characterization of CO/calcite(10.4) after geometry relaxation. The parameters $x\left(C-C a_{(\text {surface) }}\right), y\left(C-C a_{(\text {surface) }}\right)$ and $z\left(C-C a_{(\text {surface) }}\right)$ denote the distance between the carbon atom of the adsorbed $\mathrm{CO}$ molecule and the surface $\mathrm{Ca}$ ion. $\mathrm{Ca}\left({ }_{\text {surface }}\right)-\mathrm{O}$ (subsurface) indicate the distance between the surface $\mathrm{Ca}$ ion and $\mathrm{O}$ presented in the sublayer. $\Theta$ (tilt angle) and $\varphi$ (azimuthal angle) are defined based on the presented methods in Fig. 6 and 7

\begin{tabular}{|c|c|c|c|}
\hline & \multirow{2}{*}{$\frac{0.5 \mathrm{ML}}{\mathrm{CO}}$} & \multicolumn{2}{|c|}{$1.0 \mathrm{ML}$} \\
\hline & & $\mathrm{CO}$ & $(\mathrm{CO})_{2}$ \\
\hline $\mathrm{C}-\mathrm{O} / \AA$ & 1.14 & 1.14 & 1.14 \\
\hline$x\left(\mathrm{C}-\mathrm{Ca}_{(\text {surface })}\right) / \AA$ & 0.04 & 0.06 & 0.05 \\
\hline$y\left(\mathrm{C}-\mathrm{Ca}_{(\text {surface })}\right) / \AA$ & 0.02 & 0.02 & 0.02 \\
\hline$z\left(\mathrm{C}-\mathrm{Ca}_{\text {(surface) }}\right) / \AA$ & 2.78 & 2.78 & 2.79 \\
\hline $\left.\mathrm{Ca}_{(\text {surface })}\right)^{-} \mathrm{O}_{\text {(subsurface) }}$ & 2.33 & 2.33 & 2.33 \\
\hline$\Theta /(\mathrm{deg})$ & 14.42 & 14.57 & 15.14 \\
\hline$\Phi /(\mathrm{deg})$ & 36.49 & 36.18 & 38.34 \\
\hline
\end{tabular}

for the two CO molecules within the $(1 \times 1)$ unit cell at $1 \mathrm{ML}$. The optimized geometries obtained for the different configurations are depicted in Fig. 6 and 7, according parameters are shown in Table 1. The slight increase in the tilt angle with coverage reveals the influence of lateral interactions between the CO molecules. ${ }^{32}$

The calculated adsorption energies and stretching frequencies of $\mathrm{CO}$ molecules at different coverages are reported in Table 2. For the isolated CO gas phase molecule, a stretch frequency of $2123 \mathrm{~cm}^{-1}$ was obtained, showing the frequency shift $(\Delta \nu)$ of $20 \mathrm{~cm}^{-1}$ compared to the experimental value of $2143 \mathrm{~cm}^{-1}$. 32 In the following, we will use the relative shifts to the free molecule, $\Delta \nu$, when comparing experimental and theoretical results.
Table 2 Adsorption energy and vibrational frequencies as a function of surface coverage

\begin{tabular}{llllll}
\hline $\begin{array}{l}\text { CO cov- } \\
\text { erage/ }\end{array}$ & $\begin{array}{l}\Delta \nu \text { calcu- } \\
\text { lated/ } \\
\mathrm{ML}\end{array}$ & $\begin{array}{l}\nu\left(\mathrm{correc}^{-1}\right. \\
\left.\mathrm{ted}^{a}\right) / \mathrm{cm}^{-1}\end{array}$ & $\begin{array}{l}\text { Measured } \\
\text { frequency/ } \\
\mathrm{cm}^{-1}\end{array}$ & $\begin{array}{l}\text { Calculated } \\
E_{\text {ads }} / \mathrm{eV}\end{array}$ & $\begin{array}{l}\text { Measured } \\
E_{\text {ads }} / \mathrm{eV}\end{array}$ \\
\hline 0.5 & 27 & 2177 & 2175 & -0.216 & - \\
1.0 & 19 & 2161 & 2173 & -0.210 & -0.31 \\
${ }^{a}$ Corrected by PBE-scale factor: & 0.994. & & \\
\hline
\end{tabular}

For a single CO molecule at 0.5 ML coverage, the adsorption energy is calculated as $-0.216 \mathrm{eV}$, and the $\mathrm{CO}$ stretching frequency is blue shifted by $27 \mathrm{~cm}^{-1}$ relative to the gas phase value. For the full monolayer, the binding energy is lowered to $-0.210 \mathrm{eV}$ and the blue shift is reduced to $21 \mathrm{~cm}^{-1}$. The lowering of binding energy is attributed to repulsive adsorbate-adsorbate interactions, as observed for $\mathrm{CO}$ adsorption on other oxide surfaces $\left(e . g\right.$. $\left.\mathrm{TiO}_{2}{ }^{33}\right)$. The absence of attractive adsorbate-adsorbate interactions also explains why no island formation is observed when increasing exposures. A simple rationalization of the changes in frequency between low and high coverage are difficult, since the overall blue shift (relative to the free molecule) is a result of a combination of several mechanisms: (1) the interaction between the surface electric field and the CO dipole moment (Stark effect), and (2) the repulsive potential (Pauli repulsion) due to the fact that the $\mathrm{CO}$ molecule vibrates against the rigid surface (also called wall effect). ${ }^{31,33}$ Yet, intermolecular repulsive interactions are in agreement with the NC-AFM data where no clustering of the $\mathrm{CO}$ molecules is observed.

\section{Conclusions}

Polarization-resolved IRRAS and NC-AFM were used to study the adsorption of CO molecules on freshly cleaved calcite(10.4) surfaces. The NC-AFM data demonstrated a high structural quality of the surface. Adsorption of $\mathrm{CO}$ resulted in the occurrence of features appearing dark, located predominantly on-top of bright surface features. The latter were identified as the surface $\mathrm{Ca}^{2+}$ cations. The IRRAS data recorded with p-polarized incident light reveal that on the cleaved calcite(10.4) surface only one adsorbate species is present, in accord with the NC-AFM findings.

DFT calculations for CO adsorbed on the (10.4) surface of calcite were carried out for two different surface coverages (0.5 ML and 1.0 ML). According to the calculations, CO adopts a slightly tilted geometry for the two different coverages studied here. In accord with the IRRAS experiments, a blue shift of the vibrational frequencies relative to the free molecule is obtained. The difference between experimental and theoretical shifts is less than $2 \mathrm{~cm}^{-1}$ for $0.5 \mathrm{ML}$, revealing a very good agreement. For 1.0 ML the difference between experimental and theoretical shifts increases to $12 \mathrm{~cm}^{-1}$. A missing vdW-potential in the DFT functional can partially explain the discrepancy. The theoretical value for the adsorption energy of $-0.210 \mathrm{eV}$ for $1.0 \mathrm{ML}$ is good agreement with the experimental value of $-0.31 \mathrm{eV}$. The binding energy decreased for higher coverages (1.0 ML relative to $0.5 \mathrm{ML}$ ), demonstrating the presence of repulsive interactions between 
adjacent $\mathrm{CO}$ adsorbates. This observation is in accord with the NC-AFM data indicating the absence of $\mathrm{CO}$ island formation.

\section{Conflicts of interest}

The authors declare no competing financial interest.

\section{Note added after first publication}

This article replaces the version published on 09 July 2020, which contained errors in Fig. 5 and an incorrect image for Fig. 3.

\section{Acknowledgements}

PR gratefully acknowledges funding by the German Research Foundation via grant RA2832/1-1. W. W. is grateful for a postdoctoral fellowship donated by the Helmholtz Association and China Postdoctoral Council (OCPC). The results presented in this paper have been gained within the DFG-funded project TH1566/3-2. We thank Ralf Bechstein and Angelika Kühnle for providing us with a suitable sample holder.

\section{References}

1 J. W. Morse and F. T. Mackenzie, Geochemistry of sedimentary carbonates. Elsevier, 1990, vol. 48.

2 M. E. Tucker and V. P. Wright, Carbonate sedimentology. John Wiley \& Sons, 2009.

3 (a) M. S. Reddy, Biomineralization of calcium carbonates and their engineered applications: a review, Front. Microbiol., 2013, 4, 314; (b) L. Addadi and S. Weiner, Control and design principles in biological mineralization, Angew. Chem., Int. Ed. Engl., 1992, 31(2), 153-169.

4 H. Cölfen, Precipitation of carbonates: recent progress in controlled production of complex shapes, Curr. Opin. Colloid Interface Sci., 2003, 8(1), 23-31.

5 P. Hlubina and W. Urbańczyk, Dispersion of the group birefringence of a calcite crystal measured by white-light spectral interferometry, Meas. Sci. Technol., 2005, 16(6), 1267.

6 J. M. Matter and P. B. Kelemen, Permanent storage of carbon dioxide in geological reservoirs by mineral carbonation, Nat. Geosci., 2009, 2(12), 837-841.

7 (a) R. Innocenti Malini, A. R. Finney, S. A. Hall, C. L. Freeman and J. H. Harding, The Water-Amorphous Calcium Carbonate Interface and Its Interactions with Amino Acids, Cryst. Growth Des., 2017, 17(11), 5811-5822; (b) N. H. de Leeuw and S. C. Parker, Surface Structure and Morphology of Calcium Carbonate Polymorphs Calcite, Aragonite, and Vaterite: An Atomistic Approach, J. Phys. Chem. B, 1998, 102(16), 2914-2922; (c) R. Howie; J. Zussman and W. Deer, An introduction to rockforming minerals. Longman, 1992.

8 (a) N. H. de Leeuw and S. C. Parker, Surface structure and morphology of calcium carbonate polymorphs calcite, aragonite, and vaterite: an atomistic approach, J. Phys. Chem. B,
1998, 102(16), 2914-2922; (b) S. Kerisit, S. C. Parker and J. H. Harding, Atomistic simulation of the dissociative adsorption of water on calcite surfaces, J. Phys. Chem. B, 2003, 107(31), 7676-7682.

9 P. Tasker, The stability of ionic crystal surfaces, J. Phys. C: Solid State Phys., 1979, $12(22), 4977$.

10 P. Rahe, J. Schütte and A. Kühnle, NC-AFM contrast formation on the calcite (10.4) surface, J. Phys.: Condens. Matter, 2012, 24(8), 084006.

11 (a) S. Rode, N. Oyabu, K. Kobayashi, H. Yamada and A. Kühnle, True atomic-resolution imaging of (1014) calcite in aqueous solution by frequency modulation atomic force microscopy, Langmuir, 2009, 25(5), 2850-2853; (b) J. Schütte, P. Rahe, L. Tröger, S. Rode, R. Bechstein, M. Reichling and A. Kühnle, Clear signature of the $(2 \times 1)$ reconstruction of calcite (1014), Langmuir, 2010, 26(11), 8295-8300; (c) P. Fenter, P. Geissbühler, E. DiMasi, G. Srajer, L. Sorensen and N. Sturchio, Surface speciation of calcite observed in situ by high-resolution X-ray reflectivity, Geochim. Cosmochim. Acta, 2000, 64(7), 1221-1228; (d) S. L. Stipp and M. F. Hochella Jr, Structure and bonding environments at the calcite surface as observed with X-ray photoelectron spectroscopy (XPS) and low energy electron diffraction (LEED), Geochim. Cosmochim. Acta, 1991, 55(6), 1723-1736.

12 (a) S. Kerisit, A. Marmier and S. C. Parker, Ab initio surface phase diagram of the $\{1014\}$ calcite surface, J. Phys. Chem. B, 2005, 109(39), 18211-18213; (b) A. Silvestri, A. Budi, E. Ataman, M. Olsson, M. Andersson, S. Stipp, J. Gale and P. Raiteri, A Quantum Mechanically Derived Force Field To Predict $\mathrm{CO}_{2}$ Adsorption on Calcite $\{10.4\}$ in an Aqueous Environment, J. Phys. Chem. C, 2017, 121(43), 24025-24035; (c) M. P. Andersson, K. Dideriksen, H. Sakuma and S. L. S. Stipp, Modelling how incorporation of divalent cations affects calcite wettability-implications for biomineralisation and oil recovery, Sci. Rep., 2016, 6, 28854.

13 (a) G. Raina, R. Gauldie, S. K. Sharma and C. E. Helsley, A study of the calcite cleavage plane using the atomic force microscope, Ferroelectr., Lett. Sect., 1994, 17(3-4), 65-72; (b) J. Schütte, P. Rahe, L. Tröger, S. Rode, R. Bechstein, M. Reichling and A. Kühnle, Clear Signature of the $(2 \times 1)$ Reconstruction of Calcite (1014), Langmuir, 2010, 26(11), 8295-8300.

14 A. L. Rachlin, G. S. Henderson and M. C. Goh, An atomic force microscope (AFM) study of the calcite cleavage plane: image averaging in Fourier space, Am. Mineral., 1992, 77 (9-10), 904-910.

15 C. Wöll, Structure and Chemical Properties of Oxide Nanoparticles Determined by Surface-Ligand IR Spectroscopy, ACS Catal., 2019, 10(1), 168-176.

16 Y. Wang and C. Wöll, IR spectroscopic investigations of chemical and photochemical reactions on metal oxides: bridging the materials gap, Chem. Soc. Rev., 2017, 46(7), 1875-1932.

17 L. Tröger, J. Schütte, F. Ostendorf, A. Kühnle and M. Reichling, Concept for support and cleavage of brittle crystals, Rev. Sci. Instrum., 2009, 80(6), 063703. 
18 C. Barth and C. R. Henry, Kelvin probe force microscopy on surfaces of UHV cleaved ionic crystals, Nanotechnology, 2006, 17(7), S155.

19 F. J. Giessibl, High-speed force sensor for force microscopy and profilometry utilizing a quartz tuning fork, Appl. Phys. Lett., 1998, 73(26), 3956-3958.

20 L. Bartels, G. Meyer and K.-H. Rieder, Controlled vertical manipulation of single CO molecules with the scanning tunneling microscope: a route to chemical contrast, Appl. Phys. Lett., 1997, 71(2), 213-215.

21 S. Kuhn, M. Kittelmann, Y. Sugimoto, M. Abe, A. Kühnle and P. Rahe, Identifying the absolute orientation of a lowsymmetry surface in real space, Phys. Rev. B: Condens. Matter Mater. Phys., 2014, 90(19), 195405.

22 (a) G. Kresse and J. Furthmuller, Efficient iterative schemes for ab initio total-energy calculations using a plane-wave basis set, Phys. Rev. B: Condens. Matter Mater. Phys., 1996, 54(16), 11169-11186; (b) M. Izadifar, F. Königer, A. Gerdes, C. Wöll and P. Thissen, Correlation between Composition and Mechanical Properties of Calcium Silicate Hydrates Identified by Infrared Spectroscopy and Density Functional Theory, J. Phys. Chem. C, 2019, 123(17), 10868-10873.

23 G. Kresse and D. Joubert, From ultrasoft pseudopotentials to the projector augmented-wave method, Phys. Rev. B: Condens. Matter Mater. Phys., 1999, 59(3), 1758-1775.

24 (a) S. Hwang, M. Blanco and W. A. Goddard, Atomistic simulations of corrosion inhibitors adsorbed on calcite surfaces I. Force field parameters for calcite, J. Phys. Chem. B, 2001, 105(44), 10746-10752; (b) J. S. Lardge, D. M. Duffy and M. J. Gillan, Investigation of the Interaction of Water with the Calcite (10.4) Surface Using Ab Initio Simulation, J. Phys. Chem. C, 2009, 113(17), 7207-7212.
25 H. J. Monkhorst and J. D. Pack, Special points for Brillouinzone integrations, Phys. Rev. B: Condens. Matter Mater. Phys., 1976, 13(12), 5188-5192.

26 N. Giraudo, P. G. Weidler, F. Laye, M. Schwotzer, J. Lahann, C. Wöll and P. Thissen, Corrosion of Concrete by WaterInduced Metal-Proton Exchange, J. Phys. Chem. C, 2016, 120(39), 22455-22459.

27 M. Bruno, E. Bittarello, F. Massaro and D. Aquilano, The effect of impurities on the structure and energy of a crystal surface: $\mathrm{Mg}$ impurities in calcite as a case study, CrystEngComm, 2018, 20(32), 4556-4564.

28 S. Sanna, W. G. Schmidt and P. Thissen, Formation of Hydroxyl Groups at Calcium-Silicate-Hydrate (C-S-H): Coexistence of $\mathrm{Ca}-\mathrm{OH}$ and $\mathrm{Si}-\mathrm{OH}$ on Wollastonite (001), J. Phys. Chem. C, 2014, 118(15), 8007-8013.

29 P. A. Redhead, Thermal desorption of gases, Vacuum, 1962, 12(4), 203-211.

30 A. S. Foster, A. L. Shluger and R. M. Nieminen, Quantitative modelling in scanning force microscopy on insulators, Appl. Surf. Sci., 2002, 188(3-4), 306-318.

31 G. Pacchioni, A. M. Ferrari and P. S. Bagus, Cluster and band structure ab initio calculations on the adsorption of CO on acid sites of the $\mathrm{TiO}_{2}(110)$ surface, Surf. Sci., 1996, 350(1-3), 159-175.

32 J. P. P. Ramalho, F. Illas and J. R. B. Gomes, Adsorption of CO on the rutile $\mathrm{TiO}_{2}(110)$ surface: a dispersion-corrected density functional theory study, Phys. Chem. Chem. Phys., 2017, 19(3), 2487-2494.

33 M. Setvin, M. Buchholz, W. Y. Hou, C. Zhang, B. Stoger, J. Hulva, T. Simschitz, X. Shi, J. Pavelec, G. S. Parkinson, M. C. Xu, Y. M. Wang, M. Schmid, C. Wöll, A. Selloni and U. Diebold, A Multitechnique Study of $\mathrm{CO}$ Adsorption on the $\mathrm{TiO}_{2}$ Anatase (101) Surface, J. Phys. Chem. C, 2015, 119(36), 21044-21052. 